\title{
Das Potenzial der Selektivverträge nutzen - Pro
}

\author{
The Potential of Selective Contracts in Germany - Pro
}

Autoren

Wiebke Martinsohn-Schittkowski, Ulrike Sühlfleisch-Thurau, Christoph J. Tolzin

Institut

Kompetenz-Centrum für Psychiatrie und Psychotherapie der MDK Gemeinschaft und des GKV-Spitzenverbandes, Rostock

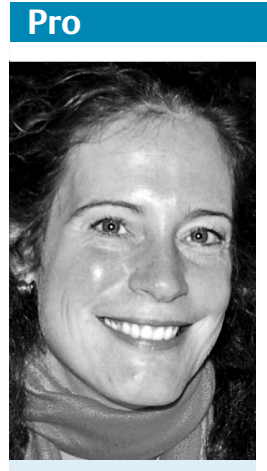

Wiebke Martinsohn-Schittkowski
Noch zu Beginn des letzten Jahrhunderts war die kollektivvertraglich organisierte ambulante ärztliche Versorgung eine Fiktion, deren Verwirklichung im Jahr 1931 als Sicherheitsnetz für die Patientenversorgung gefeiert wurde. Vorher ausschließlich im Rahmen von Einzeldienstverträgen tätig, war nunmehr jeder Vertragsarzt berechtigt und auch verpflichtet, die vertragsärztliche Versorgung zu gewährleisten.

Der diesbezüglich formulierte Sicherstellungsauftrag enthielt die ,.... bedarfsgerechte und gleichmäßige ärztliche Versorgung unter Einschluss eines ausreichenden Notdienstes..." der Versicherten und deren Angehörigen (Reichsversichertenverordnung, §368 Abs.3, Stand 1988). Heute sind die Ziele der vertragsärztlichen Versorgung in vergleichbarem Wortlaut in den verschiedenen Paragrafen des SGB V zu finden, so auch die Sicherstellung (SGB V $\S \S 70,75$ ).

Damit bildet die Orientierung am Bedarf der Versicherten auch aktuell das zentrale Element des deutschen Krankenversicherungssystems. Jedoch verfolgt die Gesundheitspolitik neben diesen normativ begründeten Zielen auch wirtschaftliche Effizienzziele, die sich zum Beispiel im Wirtschaftlichkeitsgebot des SGB V niederschlagen: „Die Leistungen müssen ausreichend zweckmäßig und wirtschaftlich sein, ..." (SGB V §12 Abs. 1).
Aus diesem Spannungsfeld erklären sich die mannigfaltigen Reformbestrebungen der letzten Jahrzehnte, über mehr wettbewerbliche Steuerung und eine Anreizregulierung die Qualität und die Wirtschaftlichkeit der deutschen Gesundheitsversorgung zu steigern ([1], S.397).

Es besteht ein breiter Konsens darüber, dass in beiden Bereichen noch unausgeschöpfte Potenziale schlummern. Diese wurden über mehrere Jahre analysiert und im aktuellen Sondergutachten des Sachverständigenrats [2] auf einen denkbar einfachen Nenner gebracht: Effizienzund Effektivitätspotenziale im Gesundheitswesen liegen einerseits dort, wo sich mit den eingesetzten Ressourcen ein höherer Nutzen erreichen lässt oder aber dort, wo sich das erreichte Nutzenniveau mit einem geringeren Ressourceneinsatz realisieren lässt.

In unserem Gesundheitswesen finden sich verschiedene denkbare Wettbewerbsfelder, die sich hinsichtlich ihrer Vertragsebenen, -partner sowie -inhalte unterscheiden. Eines davon ist das selektivvertragliche System. Es ist darüber definiert, dass Leistungserbringer miteinander um Verträge mit den Krankenkassen konkurrieren können und sollen. Jenseits der kollektiven Vereinbarungen werden die angebotenen Leistungen über den Preis oder die Qualität neu definiert.

Die rechtlichen Grundlagen für Selektivverträge sind im Sozialgesetzbuch $\mathrm{V}$ in den $\S \S 73$ b und 73 c fixiert.

Akteure dieser besonderen Form der ärztlichen Versorgung sind die Leistungserbringer selbst, die mit der jeweiligen Kasse Verträge schließen können. Das Spektrum reicht von einer medizinischen Behandlung spezifischer Indikationen bis hin zur Vollversorgung des einzelnen Patienten, solange die allgemein üblichen Qualitätsanforderungen als Mindestvoraussetzung eingehalten werden. Leistungen, die der GBA für die GKV abgelehnt hat, dürfen auch in Selektivverträgen nicht vereinbart werden.

Das Wort Selektion bezieht sich also auf die Auswahl der besten Anbieter und deren vertragliche Verpflichtung. Die Krankenkassen können über derartige Leistungsangebote gezielt und spezifisch auf die Bedürfnisse ihrer Versichertenpopulation eingehen. Sie können entweder ihre Ausgaben mit dem Fokus auf die Beitragsentwicklung niedrig halten (Preiswettbewerb) oder anstreben, über eine verbesserte Versorgung die gesundheitlichen Outcomes der Versicherten zu erhöhen (Qualitätswettbewerb).

Und auch die Patientinnen und Patienten haben einen unmittelbaren Einfluss auf das Wettbewerbsgeschehen, indem sie bestimmte Programme und Angebote aktiv wählen. Beispielsweise können sie gezielt Angebote nutzen, bei denen die Qualität am besten und/oder die Komplikationsrate gering ist.

Die Schwerpunktsetzung auf die Qualität der Gesundheitsversorgung könnte sich aus verschiedenen Gründen in Zukunft lohnen. Internationale Studien belegen, dass schon allein die Messung der Qualität eine verbesserte Gesundheitsversorgung und damit auch positive Effekte auf die gesundheitlichen Outcomes verspricht ([2], S.47). Der systematische Vergleich erbrachter Leistungen ermöglicht eine Analyse von Schwachstellen. Entsprechende Gegenmaßnahmen können ergriffen werden (Benchmark). Die nachfolgende Berichterstattung gibt Patienten, Versi- 
cherten und Krankenkassen Auskunft über die Resultate, aber auch allgemeine Informationen über die vorhandenen Leistungsqualitäten. Insbesondere bei planbaren Eingriffen bietet sich eine wertvolle Möglichkeit der Informationsbeschaffung. Durch die gezielte Wahl qualitativ ansprechender Leistungen kann wiederum die Anstrengung der Leistungserbringer erhöht werden, ihr Angebot kontinuierlich zu verbessern. Zur Erzeugung einer derartigen Qualitätstransparenz eröffnen sich bislang ungenutzte Ressourcen.

Somit sollte die Entfaltung dieser Potenziale eigentlich nicht schwierig sein. Dennoch herrschen aktuell immer noch die kollektiven Vereinbarungen zwischen den Verbänden der Krankenkassen einerseits und den Verbänden der Vertragsärzte und der Krankenhäuser andererseits vor. Wettbewerbliche Optionen bilden die Ausnahme ([2], S.65).

Die Gründe wurden in der Vergangenheit mannigfaltig diskutiert. Bislang sei zwar eine stärkere Konzentration der Krankenkassen auf den Preiswettbewerb zu beobachten, fand der Sachverständigenrat heraus ([2], S.31), was sich aber zumindest z.T. aus Defiziten im Bereich des Qualitätswettbewerbs erklären lasse. Es fehle noch immer an den Grundlagen der Qualitätsmessung. So stehe die Entwicklung zuverlässiger Indikatoren der Prozess- und Ergebnisqualität aus - Werkzeuge, die zur Bewertung von Leistungen herangezogen werden.

Weil im Vergleich zu den Kollektivverträgen die Gestaltung der Vertragsinhalte um ein Vielfaches freier ist, eröffnen sich mannigfaltige Möglichkeiten an Innovationen. Das Wort „innovativ“ ist nicht inhaltlich belegt. Damit bieten sich unterschiedlichste Gestaltungsmöglichkeiten. So etwa kann sich das Behandlungssetting im Rahmen eines Hometreatment stationsersetzend in den häuslichen Bereich verlagern. Angebote, die sich durch eine Qualität über die gesetzlichen Mindestanforderungen hinaus auszeichnen, können Grundlagen für die Verbesserung der allgemeinen Versorgung schaffen. Die Ergebnisse einer begleitenden Evaluation sind als Ausgangspunkt für die Verbesserung der allgemeinen Versorgungssituation geeignet.

Zusammenfassend sind Selektivverträge als wettbewerbliches Element in unserem ärztlichen Versorgungssystem zu begrüßen. Mit Bedacht vereinbart können sie einen Ausgangspunkt für eine optimierte Patientenversorgung bieten. Im günstigsten Fall profitieren auch die Leistungserbringer durch eine gesicherte Auftragslage und die Krankenkassen durch ein effizientes Preis-Leistungs-Verhältnis.
Durch die Verpflichtung zu einer Begleitevaluation können Selektivverträge eine wertvolle Quelle für die Beurteilung innovativer Behandlungsansätze sein.

\section{Literatur}

1 Monopolkommission. Mehr Wettbewerb, wenig Ausnahmen. Achtzehntes Hauptgutachten der Monopolkommission 2008/ 2009, Bonn: 2010

2 SACHVERSTÄNDIGENRAT zur Begutachtung der Entwicklung im Gesundheitswesen. Sondergutachten im Auftrag des Bundesministers für Gesundheit vom Dezember 20120 zum Thema „Wettbewerb an den Schnittstellen der Gesundheitsversorgung“. Bonn: 2012

3 Sozialgesetzbuch SGB. Haarfeld: 2012

\section{Korrespondenzadresse}

Dr. med. Wiebke Martinsohn-Schittkowski

Kompetenz-Centrum für Psychiatrie und Psychotherapie der MDK Gemeinschaft und des GKV-Spitzenverbandes

Blücherstraße $27 \mathrm{C}$

18055 Rostock

w.martinsohn-schittkowski@mdk-mv.de

Bibliografie

DOI http://dx.doi.org/

10.1055/s-0032-1327263

Psychiat Prax 2012; 39: 367-368

(c) Georg Thieme Verlag KG

Stuttgart · New York

ISSN 0303-4259 